\title{
Parasitoides de minadores de hojas y manejo de plagas
}

\author{
Adriana Salvo ${ }^{1}$ y Graciela R. Valladares \\ Centro de Investigaciones Entomológicas de Córdoba, Instituto Multidisciplinario de Biología Vegetal. \\ Consejo Nacional de Investigaciones Científicas y Tecnológicas. \\ Facultad de Ciencias Exactas, Físicas y Naturales. Universidad Nacional de Córdoba. \\ Av. Vélez Sarsfield 1611. X5016GCA. Córdoba, Argentina.
}

\begin{abstract}
A. Salvo, and G.R. Valladares. 2007. Leafminer parasitoids and pest management. Cien. Inv. Agr. 34(3):167-185. Leafminers are insects whose larvae live and feed within plant leaves, consuming mesophyll tissue without damaging the leaf epidermis. Several species are considered serious pests on intensive, horticultural, and ornamental crops. Natural enemies are the most frequent source of mortality for this herbivore insect guild, with parasitoids being the most effective and best represented source. This article provides an updated summary of the available research on leafminer parasitoids in relation to pest management. Parasitoids of leafminers are predominantly generalists, and can thus rapidly include in their host ranges newly introduced leafminer species, frequently achieving effective regulation a few years after the pest becomes established. Classical and augmentative biological control strategies are broadly used for leafminer pest management. Several studies have dealt with the simultaneous use of parasitoids together with chemical and cultural control. Many conventional insecticides have detrimental effects on parasitoids; however, others could be compatible with biological control. Although integrated pest management programs employing a combination of several control strategies have achieved success against leafminer pests, the effects of cultural practices that could boost parasitoid populations have been scarcely studied.
\end{abstract}

Key words: Biological control, chemical control, cultural control, leafminers, parasitoids, pest management.

\section{Introducción}

Los parasitoides son insectos de complejas y fascinantes biologías, cuyas larvas se alimentan de otros insectos, a los que causan la muerte para completar su desarrollo. Aunque pasan inadvertidos por su pequeño tamaño, este grupo de organismos posee una tremenda importancia económica, ya que actúan como reguladores poblacionales de sus hospedadores, representando herramientas útiles para el manejo de insectos plaga. Los parasitoides de minadores de hojas constituyen un grupo interesante y relativamente bien estudiado de especies pertenecientes a por lo menos diez

Recibido 07 junio 2007. Aceptado 23 julio 2007.

'Dirigir correspondencia a A. Salvo: asalvo@efn.uncor.edu familias del Orden Hymenoptera, Suborden Apocrita. Se trata de insectos que se han adaptado a las particulares condiciones de vida de sus hospedadores y tienen gran potencialidad en programas de control biológico (Hawkins et al., 1993).

En el presente artículo se incluye una revisión de los parasitoides de minadores de hojas en el contexto de su posible utilización para el manejo de especies plaga. Con este objetivo se revisó la literatura referida a aspectos ecológicos, tanto teóricos como prácticos, que se deben tener en cuenta para el empleo de parasitoides como reguladores de las poblaciones de minadores de hojas. Asimismo, se discuten otras estrategias utilizadas en el control de minadores, con énfasis en la relación entre dichos métodos y la regulación ejercida por los parasitoides. 
Previamente se introduce el tema mediante una breve caracterización de los insectos minadores de hojas, su importancia económica, las causas por las que varias de sus especies alcanzan estatus de plaga en diferentes cultivos, y la importancia relativa de los parasitoides para la regulación de sus poblaciones.

\section{Los minadores de hojas}

Los minadores de hojas son insectos cuyos estados inmaduros viven y se alimentan dentro de las hojas, consumiendoel mesófilo sin dañar la epidermis foliar. Los rastros de su alimentación ("minas") son visibles externamente en las hojas, como áreas blanquecinas o pardas y con formas variables, desde estrechas galerías lineales hasta amplias cámaras (Figura 1) (Hering, 1951). El hábito de minar hojas se ha desarrollado en un grupo de más de 10.000 especies de insectos holometábolos, concentradas en cuatro órdenes: Diptera, Coleoptera, Hymenoptera y Lepidoptera (Connor y Taverner, 1997).

Las galerías excavadas por las larvas minadoras pueden reducir la capacidad fotosintética de las hojas, causar abscisión foliar prematura y permitir el ingreso de fitopatógenos a las plantas. Además, reducen el valor estético de las plantas ornamentales o de hojas comestibles (Spencer, 1973; Parrella y Jones, 1987; Minkenberg y Van Lenteren, 1986; Maier, 2001; Valladares, en prensa). Varias especies son consideradas plagas en diversas partes del mundo. Entre éstas, el minador de los cítricos Phyllocnistis citrella Stainton (Lepidoptera: Gracillariidae) y más de 100 especies de moscas minadoras (Diptera: Agromyzidae), destacándose Liriomyza trifolii Burgess y Liriomyza huidobrensis Blanchard en cultivos hortícolas, y Agromyza frontella (Rondan) en alfalfa (Amalin, et al. 2002; Dempewolf, 2004).

La mayoría de los autores concuerdan en que una especie de minador de hojas se convierte en plaga por el desarrollo de resistencia a insecticidas y por la eliminación de sus enemigos naturales. Esta última, a consecuencia de las prácticas agrícolas agresivas (ej. aradura, roturado, quema del suelo, etc.) y al empleo de insumos químicos (Spencer, 1973; Minkenberg y Van Lenteren, 1986). Además, otros dos factores pueden contribuir decisivamente a que los minadores alcancen elevados tamaños poblacionales: 1. Relativa inconspicuidad, lo cual les permite pasar inadvertidos hasta alcanzar altas densidades (Maier, 2001), y 2. La protección que consiguen sus estados inmaduros dentro de los tejidos vegetales, especialmente contra los efectos de insecticidas de contacto. Esta última característica ha promovido el uso indiscriminado de insecticidas de amplio espectro, que han diezmado las poblaciones de enemigos naturales. Mientras tanto, los adultos minadores han desarrollado resistencia, pasando de ser plagas secundarias a primarias (Murphy y La Salle, 1999; Civelek y Weintraub, 2003). Ejemplos concretos de esta situación son Liriomyza sativae (Hills y Taylor, 1951), L. trifolii (Reitz et al., 1999), los minadores del género Phyllonorycter sobre frutales (Maier, 2001) y varias plagas de minadores en tomate (Gelenter y Trumble, 1999).

Otro factor que pudo contribuir para que ciertas especies de minadores se conviertan en plaga es el incremento de los monocultivos. Muchos parasitoides tienen preferencias por plantas específicas. Por lo tanto, si el único cultivo presente es poco atractivo para los parasitoides; en ese ambiente, los minadores pueden escapar al parasitismo (Murphy y La Salle, 1999). Finalmente, la expansión de la horticultura extensiva y el comercio de plantas sin controles cuarentenarios apropiados han propiciado también la expansión de los rangos de distribución de minadores de hojas plaga.

\section{Causas de mortalidad para los minadores de hojas}

La competencia intraespecífica, tanto directa por interferencia como indirecta o explotativa, representa una causa importante de mortalidad para las larvas minadoras de hojas (Faeth, 1990; Auerbach et al., 1995; Eber, 2004). La abscisión foliar, se ha señalado también como otro factor importante para la supervivencia de los minadores (Potter, 1985; Faeth, 1990; Girardoz et al., 2006a). La abscisión se puede interpretar como una defensa de la planta inducida por el ataque del minador. No obstante, en algunos casos, podría beneficiar a los minadores al liberarlos de sus eventuales parasitoides (Kahn 
Cuadro 1. Estudios que evalúan la toxicidad de diversas sustancias químicas frente a parasitoides de minadores de hojas. Table 1. Studies evaluating the toxicity of several chemical substances for parasitoids of leafminers.

\begin{tabular}{|c|c|c|c|}
\hline \multirow[t]{2}{*}{ Plaguicidas } & \multicolumn{3}{|c|}{ Toxicidad $^{1}$} \\
\hline & Alta & Moderada & Baja \\
\hline Aceite de $p$ & & $\begin{array}{l}\text { Conti et al.,2004 } \\
\text { Mafi and Ohbayashi, } 2006\end{array}$ & $\begin{array}{l}\text { Villanueva-Jiménez } \\
\text { and Hoy, } 1998\end{array}$ \\
\hline
\end{tabular}

Acetamiprid

Alanycarb

Abamectin

Bifenthrin

Carbosulfan

Cloropyrifos

Clothianidin

Cyromazin

Diflubenzuron

Dimetoato

Dinotefuran

Etofenprox

Fenoxycarb

Fenvalerato

Flufenoxuron

Imidacloprid

Isoxathion

Lufenuron

Mancozeb

Methomyl

Milbemectin

Neem y otros

plaguicidas naturales Tran et al., 2005

Mafi and Ohbayashi, 2006

Tran et al., 2005

Saito, 2004

Mafi and Ohbr

Saito 2004

Organofosforados Villanueva-Jiménez and Hoy, 1998

Othion

Villanueva-Jiménez and Hoy, 1998

Oxamyl

Permethrin

Pimetrozin

Profenofos

Prothiofos

Tran et al., 2005

Hidrayani et al., 2005

Saito, 2004

Teflubenzuron

Thiamethoxam

Mafi and Ohbayashi, 2006

Mafi and Ohbayashi, 2006
Hidrayani et al., 2005

Parrella and Kaspi, 2005 van Driesche et al., 1998

van Driesche et al., 1998

Conti et al., 2004

Mafi and Ohbayashi, 2006

Weintraub and Horowitz, 1998

Weintraub, 1999.

van Driesche et al., 1998

Mafi and Ohbayashi, 2006

Mafi and Ohbayashi, 2006

Weintraub, 1999; Shen et al., 2003; Prijono et al., 2004;

Bjorksten and Robinson, 2005

Villanueva-Jiménezand Hoy, 1998; Mafi and Ohbayashi, 2006

Parrella et al., 1983;

Grenier and Grenier, 1993;

Villanueva-Jiménez and

Hoy, 1998.

Rathman et al., 1990

Shen et al., 2003

Villanueva-Jiménez and Hoy, 1998.

Prijono et al., 2004;

Bjorksten and Robinson, 2005

Rathman et al., 1990

Ohno et al., 1999

Villanueva-Jiménez and

Hoy, 1998; Immaraju, 1998;

Abou-Fakhr Hammad et al., 2000; Banchio et al., 2003;

Chen et al., 2003a;

Shen et al., 2003.

Rathman et al., 1990

van Driesche et al.,1998

Rathman et al., 1990

Rathman et al., 1990

Mafi and Ohbayashi, 2006

'Dada la multiplicidad de enfoques utilizados en los estudios, se consideran tres niveles cualitativos de toxicidad: baja, los parasitoides no son significativamente afectados; alta, elevada mortalidad y/o notable reducción poblacional en parasitoides y moderada, se perciben efectos, pero éstos no son de gran magnitud o varían entre generaciones, tratamientos, etc.

${ }^{\prime}$ Given the various approaches used in the studies, three qualitative levels of toxicity were considered: low, parasitoids are not significantly affected; high, parasitoids suffer high mortality and/or population reduction; and moderated, effects are noticeable, but they are not very strong, or are variable among generations, treatments, etc. 
y Cornell, 1989). Por otra parte, algunas larvas minadoras liberan citoquininas que mantienen áreas verdes ("islas verdes") en hojas senescentes, lo cual les permite completar su desarrollo. Stiling y Simberloff (1989), luego de estudiar la mortalidad de algunas especies que inducen abscisión foliar, concluyen que este fenómeno debe verse, desde un punto de vista parsimonioso, como simple respuesta de la planta al daño del fitófago. Otros mecanismos defensivos de la planta, mayormente relacionados con aspectos químicos y físicos han sido mencionados (Valladares y Lawton, 1991).

La acción de los enemigos naturales ocurre por depredación y parasitismo. La depredación se ha citado como la mayor causa de mortalidad al comienzo de la estación de crecimiento de algunos cultivos, mientras que el parasitismo es más importante en etapas más avanzadas del desarrollo de los cultivos (Queiroz, 2002; Urbaneja et al., 2000). Excepcionalmente, para algunos minadores como Tuta absoluta (Lepidoptera: Gelechiidae), se citan valores de mortalidad por parasitismo muy bajos $(<1 \%)$ y altos niveles de mortalidad larval por predación, los que pueden alcanzar al $80 \%$ (Motta Miranda et al., 1998).

Los depredadores de minadores de hojas incluyen aves, arácnidos e insectos. Entre los insectos existen especies depredadoras en varias familias de coleópteros (ej. Carabidae, Cicindelidae, Staphylinidae), hemípteros (ej. Anthocoridae, Nabidae, Lygaeidae) e hymenopteros (ej. hormigas) (Cisneros y Mujica, 1998; Motta Miranda et al., 1998; Arno et al., 2003; Grabenweger et al., 2005).

El efecto de los depredadores a veces sobrepasa al parasitismo (Memmott et al., 1993; Queiroz, 2002; Xiao et al., 2007), incluso pueden tener un efecto adverso sobre la mortalidad que causan los parasitoides (Sato y Higashi, 1987).

Si bien la influencia relativa de depredadores y parasitoides para la regulación de las distintas especies de minadores de hojas es variable, la literatura indica que los parasitoides constituyen en general el grupo más importante (Parrella, 1987; Hespenheide, 1991). Estos tienen una función clave en el control de las poblaciones de estos insectos en ecosistemas naturales y en áreas cultivadas con uso racional de insecticidas (Lewis et al., 2002).

\section{Los parasitoides de minadores de hojas}

Los minadores de hojas conforman el gremio (grupo de organismos que consumen, de igual modo, el mismo recurso) de fitófagos con el mayor número de especies parasitoides por especie hospedadora y el que tiene las más elevadas tasas promedio de parasitismo (Hawkins, 1994). Características propias del hábito de minar hojas, tales como la escasa movilidad de las larvas, la gran visibilidad de las minas producidas y la escasa protección física proporcionada por la epidermis foliar, serían las principales causas de la vulnerabilidad de los minadores a los parasitoides (Hochberg y Hawkins, 1992). Por otra parte, los minadores de hojas son insectos herbívoros caracterizados por una marcada homogeneidad tanto ecológica como taxonómica (Connor y Taverner 1997), lo que facilitaría el desarrollo de una comunidad diversa de parasitoides compartiendo hospedadores, y explicaría la elevada carga de especies parasíticas que en conjunto tiene este grupo de insectos (Godfray, 1994).

La mayor parte de las especies de parasitoides de minadores de hojas, corresponden taxonómicamente a las superfamilias Chalcidoidea (Familias Eulophidae y Pteromalidae), Ichneumonoidea (Familia Braconidae) y Cynipoidea (Familia Figitidae) (Salvo, en prensa). Estos insectos se pueden clasificar, como idiobiontes, cuando paralizan permanentemente al hospedador en el momento de la oviposición o koinobiontes, los que paralizan sólo momentáneamente al hospedador permitiéndole continuar su desarrollo antes de provocarle la muerte (Askew y Shaw, 1986). La dicotomía idiobionte/koinobionte estaría asociada a una serie de características diferenciales, relacionadas con preferencia por grupos de hospedadores, especificidad alimenticia, estrategia reproductiva, tiempo de desarrollo, capacidad competitiva, existencia de dimorfismo sexual, etc. (Gauld y Bolton, 1988; Salvo y Valladares, 1999).

Los idiobiontes, al paralizar permanentemente 
a sus hospedadores, se exponen a que su recurso alimenticio sea atacado por otros organismos, por lo que estarían asociados con mayor frecuencia a insectos endofitófagos, que se alimentan dentro de tejidos vegetales y están mejor protegidos de condiciones desfavorables (Quicke, 1997). Por otra parte, al atacar un recurso sin defensas fisiológicas, los idiobiontes podrían consumir mayor variedad de hospedadores.

En cambio, los koinobiontes deben convivir con un organismo activo y se restringen entonces, a un menor número de especies hospedantes. Por ser insectos endófagos, se predice que los complejos parasíticos de minadores de hojas estén dominados por especies idiobiontes (Hawkins, 1994), lo cual no siempre ocurre (Salvo, 1996).

En cuanto a su especificidad alimenticia, la
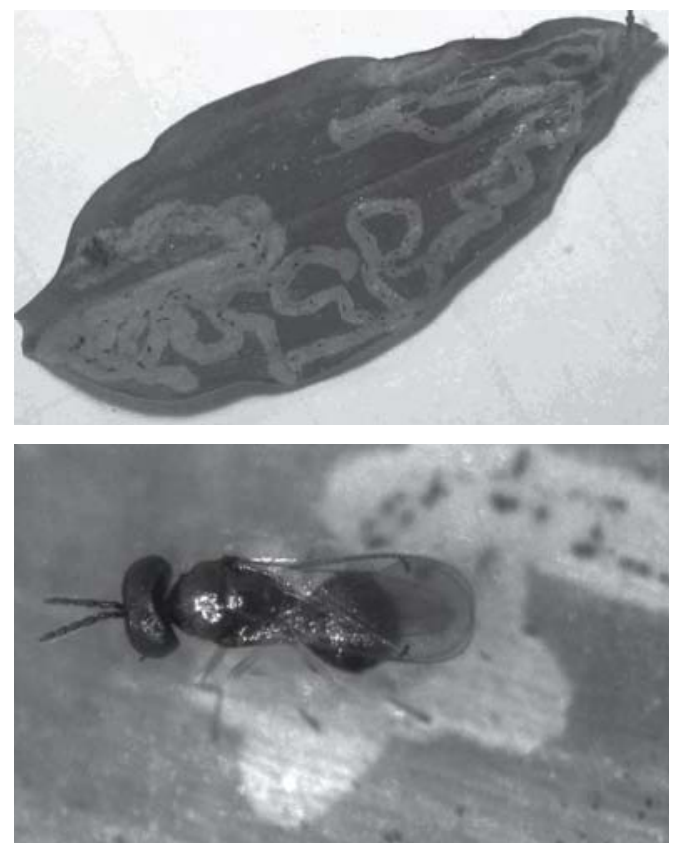

Figura 1. Minador de hojas, Liriomyza commelinae (Frost) (Diptera: Agromyzidae). A. Mina de L. commelinae (Frost) sobre una hoja de Commelina erecta $\mathrm{L}$. (Commelinaceae). B. Hembra del parasitoide Chrysocharis flacilla (Hymenoptera: Eulophidae) buscando su hospedante.

Figure 1. Leafmine of Liriomyza commelinae (Frost) (Diptera: Agromyzidae). A. Leafmine on Commelina erecta L. (Commelinaceae). B. Female parasitoid, Chrysocharis flacilla (Hymenoptera: Eulophidae) searching for its host. literatura aporta abundante evidencia de que los parasitoides de minadores de hojas poseen amplios rangos alimenticios, generalmente definidos por la ecología de sus hospedadores (Godfray, 1994; Salvo y Valladares, 1999). Esta amplitud de rango alimenticio tiene consecuencias interesantes, con respecto al manejo de especies introducidas, ya que permite a los parasitoides de minadores de hojas incorporar a las especies invasoras dentro de su espectro de hospedadores, en períodos de tiempo relativamente cortos (Murphy y La Salle, 1999). En efecto, cuando una especie de minador invade una región nueva y se convierte en plaga, posee inicialmente baja riqueza de especies parasíticas asociadas, mayormente generalistas, idiobiontes y con bajas tasas de parasitismo. No obstante, pronto es colonizada por otros parasitoides y alcanza porcentajes de parasitismo similares a los de minadores nativos, lo cual es muchas veces suficiente para lograr un control natural. Por ejemplo, este proceso se ha observado para Phyllocnistis citrella Stainton (Lepidoptera: Gracillariidae) en diversas regiones del mundo (Uygun et al., 1997; Urbaneja et al., 2000; Amalin et al., 2002; Vercher et al., 2005; Diez et al., 2006). Sin embargo, en ocasiones el reclutamiento de un complejo parasítico similar al de otros minadores no va acompañado de los niveles esperados de mortalidad (Grabenweger, 2004), lo cual se puede deber a problemas de sincronización entre la plaga y los parasitoides o a la carencia de respuestas dependientes de la densidad poblacional (densodependencia) (Malausa, 1997; Girardoz et al., 2006b).

La eficiencia de los parasitoides como agentes de mortalidad para los minadores varía según las especies. Es posible que incluso varíe entre distintos estadios larvales de una misma especie, como asimismo, según las condiciones del medio (Grabenweger, 2003). Por ejemplo, los parasitoides serían una causa de mortalidad más potente en las zonas templadas que en los trópicos (Hawkins et al., 1997; Queiroz, 2002). En forma similar, las tasas de parasitismo serían más elevadas al final de la temporada de crecimiento, al menos en sistemas cultivados (Parrella, 1987; Murphy y La Salle, 1999; Urbaneja et al., 2000). La presencia de otros organismos también puede afectar las tasas de 
parasitismo de los minadores; en este sentido, existen evidencias de efectos ocasionados por otros insectos herbívoros, como ectofítófagos masticadores (Faeth, 1985), e incluso por la presencia de hongos endofíticos (Preszler et al., 1996).

Las interacciones tritróficas planta-herbívoroparasitoide son importantes en estos sistemas (Price et al., 1980). Se ha observado que el parasitismo también puede variar en función de las especies de plantas (Olivera y Bordat 1996; Rauf y Shepard, 1999) o incluso entre cultivares o genotipos de una misma especie vegetal en que se desarrolla el minador (Fritz et al., 1997; Braman et al., 2005). Por ejemplo, en la papa, el parasitismo de L. huidobrensis es muy bajo en relación al observado en otros cultivos (Shepard et al., 1998). De acuerdo con Johnson y Hara (1987) el control biológico efectivo de ciertos minadores de hojas puede depender de la especie vegetal en la que el minador se alimenta. Un aspecto relacionado con la planta hospedante del minador que puede afectar su nivel de parasitismo, es la abscisión foliar antes mencionada. En algunos casos, esta abscisión foliar causa mortalidad tanto al minador como al parasitoide (Potter, 1985), mientras que en otros sistemas la abscisión reduce el parasitismo de las larvas debido a que los parasitoides no buscan en hojas caídas (Kahn y Cornell, 1989).

Finalmente, otros factores que afectan el parasitismo de los minadores de hojas incluyen: sequías (Staley et al., 2006), edad de la hoja (Facknath, 2005) y la posición del minador en la planta (Barrett, 1994; van der Linden 1994; Brown et al., 1997).

En los insectos parasitoides, además del parasitismo propiamente dicho, otros dos comportamientos adicionan mortalidad a los minadores de hojas: 1. Alimentación sobre el hospedador (host feeding) y 2. Paralización del hospedador sin oviposición ni alimentación (host stinging).

En el primer caso, las avispas adultas se alimentan de cierta proporción de larvas minadoras, lo cual puede o no ser condición previa imprescindible para la oviposición (Jervis y Kidd, 1986). Algunos parasitoides utilizan hospedadores de distintos tamaños, según sea para oviponer o para alimentarse (Duncan y Peña, 2000). La magnitud de la mortalidad debida a la alimentación sobre el hospedador, puede ser similar o incluso mayor que la causada por el parasitismo propiamente dicho (Amalin et al., 2002; Bernardo et al., 2006). Sin embargo, el efecto de la alimentación sobre el hospedador, funcionalmente equiparable a depredación, es frecuentemente ignorado. Esto puede subestimar notablemente los niveles de mortalidad causados por las especies de parasitoides (Cure y Cantor, 2003).

En parasitoides de minadores, particularmente en los géneros Diglyphus Walker, Sympiesis Foerster y Pnigalio Schrank (Hymenoptera, Eulophidae) se suele observar además paralización y muerte de las larvas minadoras sin que sean utilizadas para colocar huevos ni para alimentarse (Casas, 1989). Este comportamiento, interpretado como una forma de disminuir el número de larvas minadoras en la planta para asegurar la supervivencia de aquellas parasitadas, varía con el tamaño de los hospedadores disponibles, su densidad, el tamaño de las jaulas en que se crían los parasitoides y con la temperatura (Heinz and Parrella, 1989; Patel and Schuster, 1991; Patel et al., 2003).

La densodependencia en el parasitismo es un fenómeno usualmente considerado favorable para que exista una regulación efectiva de las poblaciones del hospedador (Connor and Beck, 1993; Eber et al., 2001). En algunos casos, el parasitismo causado por parasitoides nativos está más acoplado a la densidad de un minador de hojas exótico que el parasitismo causado por parasitoides introducidos para el control de la plaga (Amalin et al., 2002). La densidad de hospedadores también puede afectar otros aspectos en la relación parasitoide-minador de hojas, como ocurre con el comportamiento de búsqueda (Connor y Cargain, 1994).

\section{Control biológico}

Los casos de control biológico de minadores de hojas, citados en la literatura, dan cuenta mayormente de la introducción y liberación 
aumentativa de insectos parasitoides, aunque también han sido empleados otros organismos, tales como nemátodos y bacterias (Sher et al., 2000; van Mele y van Lenteren 2002; Cikman y Comelkcloglu, 2006). Existen numerosos ejemplos exitosos de control biológico clásico (introducción de agentes para el control de una plaga nativa o foránea) con parasitoides para distintas especies de minadores de hojas, tanto a campo abierto (Dharmadhikari et al., 1977; Johnson et al., 2003; García-Marí et al., 2004) como en invernadero (van Lenteren y Woets, 1988; Heinz y Parrella, 1990; AbdRabou, 2006). Son importantes en estos casos los estudios previos que incluyen tolerancia a la humedad, capacidad para reconocer hospedadores previamente parasitados, y hospedadores alternativos, así como la sincronización con el hospedador (Wang et al., 1999; Grabenweger, 2004; Girardoz et al., 2006b; Zappala y Hoy, 2004). También limitantes de temperatura han demostrado ser obstáculo para que un parasitoide de minadores de hojas sea introducido con éxito en algunas regiones (Klapwijk et al., 2005; Llácer et al., 2006).

La cría masiva de parasitoides para el control de minadores de hojas ha sido considerada en la literatura (Parrella et al., 1989; Kharrat y Jerraya, 2005). Diversos factores se han mencionado como importantes al momento de realizar una cría masiva, entre los que se destacan la humedad, fotoperiodo y temperatura (Yoder y Hoy 1998; Urbaneja et al., 2001; Lim et al., 2006; Kafle et al., 2005; Haghani et al., 2007).

La cría masiva de parasitoides implica el manejo simultáneo de los tres niveles tróficos, lo cual puede ser dificultoso (Smith y Hoy, 1995). Los costos y beneficios para la cría masiva de parasitoides deben ser cuidadosamente analizados, y diversas modificaciones se han planteado a partir de las técnicas convencionales para que el balance resultante sea positivo (Rizqi et al., 1999). La superproducción de machos en la cría masiva de parasitoides incrementa los costos del control biológico, porque sólo las hembras matan a los hospedadores; por ello se han desarrollado técnicas capaces de aumentar significativamente la proporción sexual de hembras mediante la utilización de hospedadores de distinto tamaño (Ode y Heinz, 2002; Chow y Heinz, 2006).

El tamaño de los minadores de hojas puede afectar no sólo la proporción de sexos sino también el tamaño de los parasitoides criados a partir de ellos, lo cual podría tener consecuencias sobre su capacidad reproductiva (Abe et al., 2005). Se han observado diferencias intraespecíficas en el tamaño corporal, tanto para parasitoides que se desarrollan en distintas especies de hospedadores (Salvo y Valladares, 1996), como para los que parasitan una misma especie de minador polífago que alcanza distintos tamaños corporales sobre distintas plantas hospedadoras (Salvo y Valladares, 2002). Estudios sobre la cría de Diglyphus websteri (Crawford) (Hymenoptera: Eulophidae) en laboratorio mostraron efectos de la temperatura, la especie hospedadora y la proporción sexual del parasitoide sobre el tamaño y otros parámetros de crecimiento de la especie criada. También se observaron complicadas interacciones entre proporción sexual-temperatura, hospedador-temperatura y hospedador-proporción sexual-temperatura (Bazzocchi et al., 2003).

Una disminución en la proporción de machos puede ser inducida mediante el uso de bacterias (Argov et al., 2000). Wolbachia es una de las bacterias más ubicuas en insectos, que manipula la reproducción del hospedador de varios modos, entre los que se puede mencionar incompatibilidad citoplasmática, muerte de machos, feminización de machos genéticos e inducción de partenogénesis (West et al., 1998). Se ha propuesto que avispas infectadas podrían ser más eficientes como agentes de control biológico de minadores de hojas (Tagami et al., 2006a,b).

Es importante tener en cuenta que los adultos obtenidos en laboratorio pueden exhibir diferencias en capacidad de parasitismo y hostfeeding en relación a adultos obtenidos a campo (Arno et al., 2003). Por lo tanto, se requieren estudios de control de calidad de los parasitoides que se obtienen mediante cría masiva.

Un largo debate, al momento de introducir enemigos naturales, consiste en conocer si una 
especie será más efectiva que varias para regular a una plaga. Algunos estudios realizados con parasitoides de minadores de hojas indican que dos especies liberadas juntas no controlan mejor al hospedador que al liberar solo una (Bader et al., 2006). Para establecer a priori la factibilidad de introducciones múltiples, se han realizado diversos estudios en laboratorio destinados a conocer el rol de la competencia y la interferencia entre parasitoides de minadores de hojas (Heimpel y Meloche, 2001; Urbaneja et al., 2003; Mitsunaga y Yano, 2004), y también la posible interacción entre predadores y parasitoides (Wu y Lin, 1998).

La introducción simultánea de parasitoides y nemátodos ha sido analizada, con resultados contradictorios. En algunos casos, las larvas del minador parasitadas se infectaron con el nemátodo, lo cual redujo la supervivencia del minador y también del parasitoide (Head et al., 2003), mientras que en otros casos, hubo compatibilidad entre parasitoides y nemátodos (Shanower et al., 1992). Un elemento decisivo podría ser el momento en que cada enemigo es liberado a campo, existiendo ejemplos en los que la aplicación de nemátodos posterior a la liberación de los parasitoides aumenta el grado de control, pero su aplicación previa es negativa (Sher et al., 2000). En el contexto de empleo simultáneo de parasitoides y otros organismos, puede considerarse también el uso de Bacillus thuringiensis, el cual ha dado buenos resultados contra algunas especies de minadores (KhyamiHorani y Ateyyat, 2002), incluso con efectos positivos sobre los parasitoides (Cikman y Comelkcloglu, 2006).

\section{Parasitoides y control químico}

En cuanto al control químico, la mayoría de los minadores de hojas son resistentes a los organofosforados, carbamatos y piretroides, y por otra parte sus enemigos naturales son severamente dañados por estos químicos, lo cual deja pocas opciones para controlarlos químicamente. Los insecticidas que no traspasan la lámina foliar son prácticamente inefectivos (Weintraub y Horowitz, 1999). Por esta razón, los insecticidas translaminares (ej. cyromacina y abamectina) son los más ampliamente utilizados contra minadores de hojas (Civelek y Weintraub, 2003). Los inhibidores de crecimiento son útiles para controlar minadores, a la vez que son potencialmente compatibles con agentes de control biológico por su baja toxicidad y alta especificidad.

Numerosos trabajos han estudiado la susceptibilidad de los parasitoides de minadores adistintos tipos de insecticidas(Tabla 1). Algunas especies de parasitoides de minadores de hojas han desarrollado resistencia, principalmente a los organofosforados. Por ejemplo, Diglyphus begini (Ashmead) (Hymenoptera: Eulophidae) es tolerante a oxamyl, methomyl, permethrin y fenvalerato (Rathman et al., 1990). El modo y el momento de aplicación del insecticida pueden afectar la susceptibilidad de los parasitoides (Kaspi y Parrella, 2005; Weintraub, 1999), la cual varía entre especies (Mafi y Ohhayashi, 2006) e, incluso, entre sexos (Rathman et al., 1992).

Los cultivos tratados con dosis bajas o sin insecticidas presentaron mayores porcentajes de parasitismo, en estudios sobre distintas especies de minadores de hojas (Galantini Vignez y Redolfi de Huiza, 1992; van Driesche et al., 1998; Adachi, 2002; Chen et al., 2003a). Igualmente, en cultivos bajo prácticas de manejo orgánicas, se han observado mayores valores de riqueza de especies parasíticas y también en su eficiencia (Balázs, 1998). Sin embargo, en otros casos no se hallaron diferencias en las densidades de parasitoides de minadores de hojas entre parcelas tratadas y no tratadas con insecticidas sintéticos (Mafi y Ohbayashi, 2004).

\section{Parasitoides y control cultural}

Es posible aumentar la acción de enemigos de minadores de hojas a través del manejo del hábitat (Price y Harbaugh, 1981). Distintos trabajos mencionan la importancia de los parches de malezas, cercanos a los cultivos, como posibles reservorios de parasitoides (Murphy y La Salle, 1999). Por esta razón se ha sugerido que el manejo de malezas y otras plantas en el agroecosistema o en el borde del mismo puede mejorar la disponibilidad de polen y néctar para los enemigos naturales de minadores de hojas (van Mele y van Lenteren, 
2002). En algunos casos, la presencia de hábitats aledaños a los cultivos con plantas en floración determinaron un aumento del parasitismo de los minadores (Chen et al., 2003b). Sin embargo, en otros casos, no se observó efecto alguno del aumento de la diversidad vegetal sobre los minadores o sus parasitoides (Johnson y Mau, 1986; Letourneau, 1995).

$\mathrm{Si}$ bien algunas malezas pueden actuar como reservorios de minadores plaga (Smith y Hardman, 1986; Schuster et al., 1991), otras albergan a minadores especialistas, no perjudiciales al cultivo. En este último caso, las malezas proveerían hospedantes alternativos para los parasitoides, aumentando el control biológico en el cultivo. Estas especies vegetales se podrían emplear para la implementación de crías abiertas de parasitoides (Parkman et al., 1989), consistentes en favorecer la presencia de plantas y minadores no perjudiciales, en el mismo ambiente del cultivo, para incrementar las poblaciones de enemigos naturales. Las crías abiertas de parasitoides han sido exitosas para algunos minadores de hojas (van der Linden, 1992). En este sentido, conocer los rangos de hospedadores de los minadores y sus parasitoides es fundamental (Parkman et al., 1989; Chen et al., 2003b; Rizzo, 2003). En algunos casos el estudio de tramas tróficas en distintos ambientes ha posibilitado la propuesta teórica de sistemas de cría abierta, contemplando las posibles interacciones entre las especies a tres niveles tróficos (Valladares y Salvo, 1999).

Los policultivos y la siembra de especies vegetales adicionales al cultivo principal pueden también tener un efecto benéfico sobre los parasitoides. Por ejemplo, se ha observado que al inicio del ciclo del cultivo de batata, las plantas de frejol sembradas en fajas en sus adyacencias, funcionan como atractivo de los parasitoides de Liriomyza huidobrensis, anticipando y aumentando su presencia en el cultivo de batata (Da Paixão Pereira et al., 2002). Asimismo, la papa cultivada con el trigo es menos dañada por los minadores, debido a que esta última especie atrae a los parasitoides (Ebwongu et al., 2001).

La suplementación de alimento para adultos de parasitoides, mediante soluciones azucaradas o miel, es una práctica de control biológico conservativo que ha sido repetidamente utilizada (Powell, 1986). Sin embargo, en algunos casos, la provisión de alimento para los parasitoides de una plaga primaria puede influir negativamente en el control de plagas secundarias, a las que generalmente pertenecen los minadores de hojas (Mitsunaga et al., 2006).

Entre las técnicas de control biológico que aumentan y conservan los enemigos naturales de minadores de hojas, se han diseñado dispositivos físicos que permiten el escape de los parasitoides, que generalmente son de tamaño más pequeño que sus hospedadores, a partir de contenedores con hojas minadas (Kehrli et al., 2005). Estos instrumentos sirven para aumentar o conservar poblaciones de parasitoides locales o foráneas y pueden ser una alternativa de bajo costo en comparación a la liberación de enemigos naturales criados convencionalmente. Este método puede ser usado cuando el control químico está prohibido, como en el caso de la agricultura orgánica. Haciendo ajustes en el tamaño de la malla, diversos sistemas de parasitoides y hospedadores pueden ser manejados de este modo (Kehrli y Bacher, 2004).

Es importante considerar que si bien algunas prácticas culturales, recomendadas para el control de insectos minadores, favorecen el aumento de las poblaciones de parasitoides, otras los perjudican. Por ejemplo, la inundación del terreno para ahogar las pupas de ciertos minadores, o la exposición de pupas a la luz solar para provocar su muerte reducen también las poblaciones de parasitoides larvopupales (Braun y Shepard, 1997).

La aplicación de compost en el cultivo puede tener un efecto indirecto sobre los minadores de hojas, aumentando la diversidad y eficacia de sus depredadores (Brown y Tworkoski, 2004). Sin embargo, los fertilizantes nitrogenados se deben usar con cuidado, ya que pueden estimular el desarrollo de las plagas, aumentando no sólo el vigor de la planta sino también la supervivencia de larvas minadoras y su tamaño pupal (van Lenteren y Overholt, 1994). Pueden existir umbrales de fertilización 
por encima de los cuales se aumenta el número de pupas del minador y disminuye el parasitismo, como se evidenció con el minador de los cítricos (Ateyyat y Mustafa, 2001). Por otra parte, niveles de nitrógeno elevados por fertilización en cultivos de frejol disminuyeron el tiempo de desarrollo y aumentaron la fertilidad del parasitoide Chrysocharis oscidinis (Hymenoptera: Eulophidae) sobre el minador Liriomyza trifolii (Kaneshiro y Johnson, 1996). En otros trabajos se ha evaluado el efecto de la fertilización sobre las tasas de parasitismo, sin analizar los mecanismos involucrados (Yarnes y Boecklen, 2006).

Una práctica recomendada para reducir las poblaciones de minadores de hojas, es la destrucción de los residuos de la planta de las cosechas anteriores que fueron atacadas, que pueden ser quemadas o enterradas (Larraín, 2004). No obstante, esta práctica también reduce las poblaciones de parasitoides (Vincent et al., 2004). Sin embargo, una poda durante el verano colocando las ramas cortadas bajo los árboles, resultó en una disminución en el número de larvas y pupas del minador de los cítricos sin efectos sobre el parasitismo (Ateyyat y Mustafa, 2001).

Las trampas pegajosas, consistentes en soportes pegajosos, generalmente de color amarillo, poseen una doble función de monitoreo de la población de minadores (conocer el momento adecuado para aplicar algún tipo de control), y también para disminuir el número de minadores en un campo (Larraín, 2004). Estas trampas son utilizadas en programas de manejo integrado de moscas minadoras (Agromyzidae), conociéndose en cierto detalle el número y superficie de trampas que deben ser colocadas para que el control sea efectivo (Braun y Shepard, 1997). Sin embargo, existe la posibilidad de que las trampas amarillas disminuyan las poblaciones de parasitoides (Gonçalves, 2006). La combinación de trampas amarillas con sustancias atractivas para los minadores, tales como extractos de hoja de la planta hospedadora, disminuye notablemente la captura de otros insectos y posiblemente también reduce el número de parasitoides allí atrapados (Harand et al., 2004).

\section{Manejo integrado de plagas y minadores}

La integración de distintas prácticas en programas de manejo integrado de plagas ha demostrado ser exitosa en el control de minadores de hojas. El reemplazo de plaguicidas químicos de síntesis por bioplaguicidas (ej. Bacillus thuringiensis) o el uso de insecticidas selectivos de bajo impacto sobre los enemigos, la disminución de los disturbios impuestos en el sistema (mediante prácticas culturales menos agresivas) y la implementación de los diversos tipos de control biológico aumentan las chances de controlar a los minadores de hojas (Murphy y La Salle, 1999).

Experimentos en jaulas en laboratorio demostraron que la liberación de Diglyphus isaea junto con la liberación de machos de $L$. huidobrensis estériles constituyen un método más eficiente que el uso de cada técnica por separado (Kaspi y Parrella, 2006), habiéndose observado que algunos parasitoides pueden desarrollarse en huevos puestos por hembras esterilizadas con rayos gamma incluso durante varias generaciones (Harwalkar et al., 1987).

Diversos trabajos abordan la implementación de programas de manejo integrado de plagas para el control de minadores, incluyendo modelos, análisis minuciosos de costos y beneficios (Dudley et al., 1989; Shepard et al., 1998; Gelernter y Trumble, 1999; Reitz et al., 1999; Motta Miranda et al., 2005), y coincidiendo en que se logra disminuir notoriamente el porcentaje de plaguicidas aplicados en relación a las aplicaciones calendario. Por ejemplo, los programas de manejo integrado de Liriomyza huidobrensis en el Perú, incluyen el uso de variedades resistentes o tolerantes, manejo del riego, eliminación de residuos de la cosecha, trampas amarillas pegajosas, insecticidas de baja toxicidad y control biológico (Palacios et al., 1995). Eninvernadero, laaplicación de adulticidas (ej. piretroides) para reducir las poblaciones iniciales del minador plaga, en combinación con dos o tres aplicaciones de insecticidas selectivos, como azadiractina, minimiza la posibilidad de resistencia y asegura el control de todos los estados de la plaga sin impedir que actúen los enemigos naturales, ampliando la ventana de control (Immaraju, 1998). 


\section{Conclusión}

A modo de conclusión, es importante remarcar que se encuentran abundantes registros bibliográficos que detallan la relación entre parasitoides y minadores de hojas. Estos consideran aspectos biológicos descriptivos o aspectos ecológicos sin relación directa con el manejo de especies plaga, trabajos que no fueron incluidos por no coincidir con el objetivo de esta revisión. También se encuentran disponibles numerosos estudios prácticos que analizan la compatibilidad del uso de parasitoides con distintos plaguicidas químicos, según se detalló anteriormente.

Los campos menos desarrollados son los que exploran la importancia de las distintas fuentes de mortalidad en tablas de vida de minadores de hojas y el impacto de las prácticas culturales sobre la fauna de parasitoides. En este sentido, prácticamente se desconoce la incidencia de las malezas aledañas al cultivo sobre los parasitoides, así como es escasa la información referida a la interacción de los parasitoides de la plaga con otros hospedadores en el agroecosistema. Disponer de este tipo de información, particularmente incorporando información sobre los tres niveles tróficos involucrados: plantas (cultivo y vegetación espontánea), minadores (plaga y hospedantes alternativos) y parasitoides, posibilitaría la implementación de estrategias de manejo ecológicamente aceptables. Sistemas de cría abierta de parasitoides, manejo de la diversidad vegetal y otras modalidades de manipulación ambiental, constituyen, en este sentido, opciones hasta ahora poco explotadas para el control de minadores plaga.

\section{Resumen}

Los minadores de hojas son insectos cuyas larvas viven y se alimentan dentro de las hojas, consumiendo el mesófilo sin dañar la epidermis foliar. Varias especies son consideradas serias plagas de cultivos intensivos, hortícolas y ornamentales. Entre las fuentes de mortalidad más importantes para este gremio de fitófagos se citan a los enemigos naturales, de los que se destacan los parasitoides como el grupo más efectivo y mejor representado. Este artículo proporciona un resumen actualizado de la información disponible sobre parasitoides de minadores de hojas en relación al manejo de plagas. Por ser generalistas, los parasitoides de minadores de hojas pueden incluir rápidamente en su rango alimenticio a especies introducidas, muchas veces lográndose un control efectivo luego de unos pocos años de establecida la plaga. Control biológico clásico y aumentativo son estrategias ampliamente usadas para regular las poblaciones de minadores de hojas plaga. Numerosos estudios abordan la compatibilidad del uso de parasitoides con control químico y cultural. Si bien la mayoría de los insecticidas convencionales poseen efectos adversos para los parasitoides, otros serían compatibles con el control biológico. Se conoce que la combinación de diversas estrategias de control en programas de manejo integrado de plagas ha resultado efectivo contra minador de hojas plaga. Sin embargo, los efectos de prácticas culturales que podrían favorecer las poblaciones de parasitoides han sido escasamente estudiados.

Palabras clave: Control biológico, control cultural, control químico, minadores de hojas, parasitoides.

\section{Agradecimientos}

Queremos expresar nuestro agradecimiento a las siguientes instituciones que han apoyado el desarrollo de estas investigaciones: CONICET, SECyT, FONCyT. Agradecemos a W. A. Quispe Avalos por la lectura del manuscrito original y a M. S. Fenoglio (Centro de Investigaciones Entomológicas, Universidad Nacional de Córdoba) por el trabajo fotográfico.

\section{Literatura citada}

Abd-Rabou S. 2006. Biological control of the leafminer, Liriomyza trifolii by introduction, releasing, evaluation of the parasitoids Diglyphus isaea and Dacnusa sibirica on vegetables crops in greenhouses in Egypt. Archives of Phytopathology and Plant Protection 39:439443.

Abe, Y., T. Takeuchi, S. Tokumaru, and J. Kamata. 2005. Comparison of the suitability of three pest leafminers (Diptera: Agromyzidae) as hosts for the parasitoid Dacnusa sibirica (Hym.: Braconidae). Eur. J. Entomol. 102:805-807. 
Abou-Fakhr Hammad, E.M., N.M. Nemer, and N.S. Kawar. 2000. Efficacy of the Chinaberry tree (Meliaceae) aqueous extracts and certain insecticides against the pea leafminer (Diptera: Agromyzidae). J. Agr. Sci. 134:413-420.

Adachi, I. 2002. Evaluation of generational percent parasitism on Lyonetia clerkella (Lepidoptera: Lyonetiidae) larvae in peach orchards under different management intensity. Appl. Entomol. Zool. 37:347-355.

Amalin, D.M., J.E. Peña, R.E. Duncan, H.W. Browning, and R. Mcsorley. 2002. Natural mortality factors acting on citrus leafminer, Phyllocnistis citrella, in lime orchards in South Florida. BioControl 47:327-347.

Argov, Y., Y. Gottlieb, S. Amin-Spector, and E. Zchori Fein. 2000. Possible symbiont-induced thelytoky in Galeopsomyia fausta, a parasitoid of the citrus leafminer Phyllocnistis citrella. Phytoparasitica 28:212-218.

Arno, J., E. Alonso, and R. Gabarra. 2003. Role of the parasitoid Diglyphus isaea (Walker) and the predator Macrolophus caliginosus Wagner in the control of leafminers. Bulletin OILB/SROP 26:79-84.

Askew, R.R., and M.R. Shaw. 1986. Parasitoid Communities: their size, structure and development. p. 225-264. In: J. Waage and D. Greathead (eds.). Insect Parasitoids. Academic Press, London.

Ateyyat, M.A., and T.M. Mustafa. 2001. Cultural control of citrus leafminer, Phyllocnistis citrella Stainton (Lepidoptera: Gracillariidae) on lemon in Jordan. Int. J. Pest Manage. 47:285-288.

Auerbach, M.J., E.F. Connor, and S. Mopper. 1995. Minor miners and major miners: population dynamics of leafmining insects. p. 83-110. In: N. Cappuccino and P.W. Price (eds.). Population Dynamics. Academic, San Diego, California.

Bader, A.E., K.M. Heinz, R.A. Wharton, and C.E. Bográn. 2006. Assessment of interspecific interactions among parasitoids on the outcome of inoculative biological control of leafminers attacking chrysanthemum Biological Control 39:441-452.

Bala'zs, K. 1998. The importance of parasitoids in apple orchards Biol. Agric. Hortic. 15:123-129.

Banchio, E., G.R. Valladares, M. Defagó, S. Palacios, and C. Carpinella. 2003. Effects of Melia azedarach (Meliaceae) fruit extracts on the leafminer Liriomyza huidobrensis (Diptera: Agromyzidae): assessment in laboratory and field experiments. Ann. Appl. Biol. 143:187-193.

Barrett, B.A. 1994. Within-tree distribution of Phyllonorycter blancardella (F.) and $P$. crataegella (Clemens) (Lepidoptera: Gracillariidae) and associated levels of parasitism in commercial apple orchards. Biol. Control 4:74-79.

Bazzocchi, G.G., A. Lanzoni, G. Burgio, and M.R. Fiacconi. 2003. Effects of temperature and host on the pre-imaginal development of the parasitoid Diglyphus isaea (Hymenoptera: Eulophidae). Biol. Control 26:74-82.

Bernardo, U., P.A. Pedata, and G. Viggiani. 2006. Life history of Pnigalio soemius (Walker) (Hymenoptera: Eulophidae) and its impact on a leafminer host through parasitization, destructive host-feeding and host-stinging behavior. Biol. Control 37:98-107.

Bjorksten, T.A., and M. Robinson. 2005. Juvenile and sublethal effects of selected pesticides on the leafminer parasitoids Hemiptarsenus varicornis and Diglyphus isaea (Hymenoptera: Eulophidae) from Australia. J. Econ. Entomol. 98:1831-1838.

Braman, S.K., G.D. Buntin, and R.D. Oetting. 2005. Species and cultivar influences on infestation by and parasitism of a columbine leafminer (Phytomyza aquilegivora Spencer). J. Environ. Hortic. 23:9-13.

Braun, A.R., and M. Shepard. 1997. Leafminer Fly: Liriomyza huidobrensis. Technical Bulletin. International Potato Center and Clemson University Palawija IPM Project. The International Potato Center. www.eseap. cipotato.org/file-library.htm (Consultado el 2 de Julio, 2007).

Brown, M.W., and T. Tworkoski. 2004. Pest management benefits of compost mulch in apple orchards. Agr. Ecosys. Environ. 103:465-472.

Brown, J.L., S. Vargo, E.F. Connor and M.S. Nuckols. 1997. Causes of vertical stratification in the density of Cameraria hamadryadella. Ecol. Entomol. 22:16-25.

Casas, J. 1989. Foraging behaviour of a leafminer parasitoid in the field. Ecol. Entomol. 14:257265.

Cikman, E., and N. Comelkcloglu. 2006. Effects of Bacillus thuringiensis on larval serpentine leafminers Liriomyza trifolii (Burgess) (Diptera: Agromyzidae) in bean. Pakistan J. Biol. Sci. 9:2082-2086.

Cisneros, F., and N. Mujica. 1998. The leafminer fly in potato: plant reaction and natural enemies as natural mortality factors. CIP Program Report. Lima Perú. http:/www.cipotato.org/Market/ PgmRprts/pr97-98/16leafmi.pdf (Accessed 30 June, 2007).

Civelek, H.S., and P.G. Weintraub. 2003. Effects of bensultap on larval serpentine leafminers, Liriomyza trifolii (Burgess) (Diptera: Agromyzidae), in tomatoes. Crop Prot. 22:479483. 
Connor, E.F., and M.J. Cargain. 1994. Densityrelated foraging behaviour in Closterocerus tricinctus, a parasitoid of the leaf-mining moth, Cameraria hamadryadella. Ecol. Entomol. 19:327-334.

Connor, E.F., and M.P. Taverner. 1997. The evolution and adaptive significance of the leaf-mining habit. Oikos 79:6-25.

Connor, E.F., and M.W. Beck. 1993. Density related mortality in Cameraria hamadryadella (Lepidoptera: Gracillariidae) at epidemic and endemic densities. Oikos 66:515-525.

Conti, F., R. Fisicaro, A. Lo Genco, S. Colazza, and G. Liotta. 2004. An IPM program for Phyllocnistis citrella Stainton in Sicily Citrus Nurseries. p. 8. Proceedings I International Cameraria Symposium Cameraria ohridella and other invasive leaf-miners in Europe. Prague, Checoslovaquia.

Cure, J.R., and F. Cantor. 2003. Atividade predadora e parasítica de Diglyphus begini (Ashm.) (Hymenoptera: Eulophidae) sobre Liriomyza huidobrensis (Blanch.) (Diptera: Agromyzidae) em cultivos de Gypsophila paniculata L. Neotrop. Entomol. 32:85-89.

Chen, A.D., Z.Q. Chen, K.J. Luo, and S. Miao. 2003a. Effects of some insecticides on field population fluctuation and parasitism of the leafminer parasitoid. J. Yunnan Agric. Univ. 18:249-252.

Chen, X., F. Lang, Z. Xu, J. He, and Y. Ma. 2003b. The occurrence of leafminers and their parasitoids on vegetables and weeds in Hangzhou area, Southeast China BioControl 48:515-527.

Chow, A., and K.M. Heinz. 2006. Control of Liriomyza langei on chrysanthemum by Diglyphus isaea produced with a standard or modified parasitoid rearing technique. J. Appl. Entomol. 130:113-121.

Da Paixão Pereira, D.I., J.C. De souza, L.V. Costa santa-cecília, P. Rebelles Reis, and M. De Abreu Souza. 2002. Parasitismo de larvas da moscaminadora Liriomyza huidobrensis Blanchard (Diptera: Agromyzidae) pelo parasitóide Opius sp. (Hymenoptera: Braconidae) na cultura da batata com faixas de feijoeiro intercaladas. Ciência e Agrotécnica, Lavras. 26:955-963.

Darvas, B.A., and A.B. Andersen. 1999 Effects of cyromazine and dimethoate on Chromatomyia fuscula (Zett.) (Dipt., Agromyzidae) and its hymenopterous parasitoids. Acta Phytopathol. Hun. 34:231-239.

Dempewolf, M. 2004. Arthropods of Economic Importance: Agromyzidae of the World. Multimedia interactive software. ETI, Amsterdam, The Netherlands.

Dharmadhikari, P.R., P.A.C.R. Perera, and T.M.F.
Hassen. 1977. A short account of the biological control of Promecotheca cumingi [Col.: Hispidae] the coconut leaf-miner, in Sri Lanka. BioControl 22: 3-18

Diez, P.A., J.E. Peña, and P. Fidalgo. 2006. Population dynamics of Phyllocnistis citrella (Lepidoptera: Gracillariidae) and its parasitoids in Tafí Viejo, Tucumán, Argentina. Fla. Entomol. 89:328335.

Dudley, N.J.A., R.A.E. Mueller, and J.A. Wightman. 1989. Application of dynamic programming for guiding IPM on groundnut leafminer in India. Crop Prot. 8:349-357.

Duncan, R., and J.E. Peña. 2000. Fecundity, host stage preferences and the effects of temperature on Pnigalio minio (Hymenoptera: Eulophidae), a parasitoid of Phyllocnistis citrella (Lepidoptera: Gracillariidae). P. Fl. St. Hortic. Soc. 113:20-24.

Eber, S., H.P. Smith, R.K. Didham, and H.V. Cornell. 2001. Holly leaf-miners on two continents: what makes an outbreak species? Ecol. Entomol. 26:124-132.

Eber, S. 2004. Bottom-up density regulation in the holly leaf-miner Phytomyza ilicis. J. Anim. Ecol. 73:948-958.

Ebwongu, M., E. Adipala, S. Kyamanywa, C.K. Ssekabembe, and A.S. Bhagsari. 2001. Influence of spatial arrangements in maize/Solanum potato intercrops on incidence of potato aphids and leaf hoppers in Uganda. Afr. Crop Sci. J. 9:175-184

Facknath, S. 2005. Leaf age and life history variables of a leafminer: the case of Liriomyza trifolii on potato leaves Entomol. Exp. Appl. 115:79-87.

Faeth, S.H. 1985. Host leaf selection by leaf miners: interactions among three trophic levels. Ecology 66:870-875.

Faeth, S.H. 1990. Aggregation of a leafminer, Cameraria sp. nov. (Davis): consequences and causes. J. Anim. Ecol. 59:569-586.

Fritz, R.S., S.E. McDonough, and A.G. Rhoads. 1997. Effects of plant hybridization on herbivoreparasitoid interactions. Oecologia 110: 360-367

Galantini Vignez, L., and I. Redolfi de Huiza. 1992. Niveles de infestación y parasitismo de Liriomyza huidobrensis en papa cultivada sin aplicación de insecticida. Rev. Per. Entomol. 35:101-106.

García-Marí, F., R. Vercher, J. Costa-Comelles, C. Marzal, and M. Villalba. 2004. Establishment of Citrostichus phyllocnistoides (Hymenoptera: Eulophidae) as a biological control agent for the citrus leafminer Phyllocnistis citrella (Lepidoptera: Gracillariidae) in Spain. Biol. Control 29:215-226.

Gauld, I., and B. Bolton. 1988. The Hymenoptera. 
British Natural History Museum. Oxford University Press. Oxford. 332 pp.

Gelernter, W.D., and J.T. Trumble. 1999. Factors in the success and failure of microbial insecticides in vegetable crops. Int. Pest Manag. Rev. 4:301-306.

Girardoz, S., M. Kenis, and D.L.J. Quicke. 2006a. Mortality factors affecting the different developmental stages of Cameraria ohridella Deschka and Dimić in Switzerland. p. 11. In: InternationalCameraria Symposium Cameraria ohridella and other invasive leaf-miners in Europe Prague, Checoslovaquia.

Girardoz, S., M. Kenis, and D.L.J. Quicke. 2006b. Recruitment of native parasitoids by an exotic leaf miner, Cameraria ohridella: hostparasitoid synchronisation and influence of the environment. Agr. For. Entomol. 8:48-56.

Godfray, H.C.J. 1994. Parasitoids: Behavioural and Evolutionary Ecology. Princeton University Press. Chichester. USA. 473 pp.

Gonçalves, M.A.A.C. 2006. Utilizacao de armadilhas cromotropicas na monitorizacao de Liriomyza spp. (Diptera: Agromyzidae) e dos seus parasitoides, no feijao verde. Boletín de Sanidad Vegetal, Plagas. 32:169-174.

Grabenweger, G. 2003. Parasitism of different larval stages of Cameraria ohridella. BioControl 48:671-684.

Grabenweger, G. 2004 Why are native european parasitoids not able to control the Horse chestnut leafminer? 1st International Cameraria Symposium Cameraria ohridella and other invasive leaf-miners in Europe Prague, Checoslovaquia. pp. 12.

Grabenweger, G., P. Kehrli, B. SchlickSteiner, F. Steiner, M. Stolz, and S. Bacher. 2005. Predator complex of the horse chestnut leafminer Cameraria ohridella: identification and impact assessment. J. Appl. Entomol. 129:353-362.

Grenier, S., and A.M. Grenier. 1993. Fenoxycarb, a fairly new Insect Growth Regulator: a review of its effects on insects. Ann. Appl. Biol. 122:369403.

Haghani, M., Y. Fathipour, A.A. Talebi, and V. Baniameri. 2007. Temperature-dependent development of Diglyphus isaea (Hymenoptera: Eulophidae) on Liriomyza sativae (Diptera: Agromyzidae) on cucumber. J. Pest Sci. 80:71-77.

Harand, W., M. Stolz, and F. Hadacek. 2004. Female mass trapping: a contribution to Cameraria ohridella control. p. 11. In: International Cameraria Symposium Cameraria ohridella and other invasive leaf-miners in Europe. Prague. Checoslovaquia.

Harwalkar, M.R., H.D. Rananavare, and G.W. Rahaikar. 1987. Development of Trichogramma brasiliensis [Hym: Trichogrammatidae] on eggs of radiation sterilized females of potato tuberworm, Phthorimaea operculella [Lep.: Gelechiidae]. BioControl 32:159-162.

Hawkins, B.A. 1994. Pattern and process in hostparasitoid interactions. Cambridge University Press, Cambridge, UK. 190 pp.

Hawkins, B.A., H.V. Cornell, and M.E. Hochberg. 1997. Predators, parasitoids, and pathogens as mortality agents in phytophagous insect populations. Ecology 78:2145-2152.

Hawkins, B.A., M. Thomas, and M.E. Hochberg. 1993. Refuge theory and classical biological control. Science 262:1429-1432.

Head, J., L.F. Palmer, and K.F.A. Walters. 2003. The compatibility of control agents used for the control of the South American leafminer, Liriomyza huidobrensis. Biocontrol Sci. Techn.13:77-86.

Heimpel, G.E., and F. Meloche. 2001 Biological control of alfalfa blotch leafminer (Diptera: Agromyzidae) in Ontario: Status and ecology of parasitoids (Hymenoptera: Braconidae, Eulophidae) 20 years after introduction. Great Lakes Entomol. 34:17-26.

Heinz, K.M., and M.P. Parrella. 1989. Attack behavior and host size selection by Diglyphus begini on Liriomyza trifolii in chrysanthemum. Entomol. Exp. Appl. 53:147-156.

Heinz, K.M., and M.P. Parrella. 1990. Biological control of insect pests on greenhouse marigolds. Environ. Entomol. 19:825-835.

Hering, E.M. 1951. Biology of the Leaf Miners. Dr. W. Junk, The Hague. The Netherlands 420 pp.

Hespenheide, H.A. 1991. Bionomics of leaf-mining insects. Annu. Rev. Entomol. 36:535-560.

Hidrayani, Purnomo, A. Rauf, P. Ridland, and A. Hoffmann. 2005. Pesticide applications on Java potato fields are ineffective in controlling leafminers, and have antagonistic effects on natural enemies of leafminers. Int. J. Pest Manag. 51:181-187.

Hills, O.A., and E.A. Taylor. 1951. Parasitization of dipterous lef-miners in cantaloups and lettuce in the Salt River Valley, Arizona. J. Econ. Entomol. 44:759-767.

Hochberg, M.E., and B.A. Hawkins. 1992. Refuges as a predictor of parasitoid diversity. Science 255:973-976.

Immaraju, J.A. 1998. The commercial use of azadirachtin and its integration into viable pest control programmes. Pestic. Sci. 54:285-289.

Jervis, M.A., and N.A.C. Kidd. 1986. Host-feeding strategies in hymenopteran parasitoids. Biol. Rev. 61:395-434.

Johnson, M.W., and H. Hara. 1987. Influence of host crop on parasitoids (Hymenoptera) of Liriomyza spp. (Diptera: Agromyzidae). Environ. Entomol. 16:339-344. 
Johnson, M.W., and R. F. L. Mau. 1986. Effects of intercropping beans and onions on populations of Liriomyza spp. and associated parasitic Hymenoptera. P. Hawaii Entomol. Soc. 27:95-103.

Johnson, S.J.D., C. Henne, and W.J. Bourgeois. 2003. Biological control of the citrus leafminer with Ageniaspis citricola (Hymenoptera: Encyrtidae) in Louisiana. Proc. Fla. State Hort. Soc. 116:224-226.

Kafle, L., P.Y. Lai, and Y.F. Chang. 2005. Functional response of a parasitoid Ganaspidium utilis (Hymenoptera: Eucoilidae) on the leafminer Liriomyza trifolii (Diptera: Agromyzidae). Insect Sci. 12:381-385.

Kahn, D.M., and H.V. Cornell. 1989. Leafminers, early leaf abscission and parasitoids: a tritrophic interaction. Ecology 70:1219-1226.

Kaneshiro, L.N., and M.W. Johnson. 1996. Tritrophic Effects of Leaf Nitrogen on Liriomyza trifolii (Burgess) and an associated parasitoid Chrysocharis oscinidis (Ashmead) on Bean. Biol. Control 6:186-192.

Kaspi, R., and M.P. Parrella. 2005. Abamectin compatibility with the leafminer parasitoid Diglyphus isaea. Biol. Control 35:172-179.

Kaspi, R., and M.P. Parrella. 2006. Improving the biological control of leafminers (Diptera: Agromyzidae) using the sterile insect technique. J. Econ. Entomol. 99:1168-1175.

Kehrli, P., and S. Bacher. 2004. Mass-hatching devices: a new biocontrol technique to augment parasitoids.p. 118-121 IV California Conference on Biological Control. Berkeley, California, USA.

Kehrli, K., M. Lehmann, and S. Bacher. 2005. Massemergence devices: a biocontrol technique for conservation and augmentation of parasitoids. Biol. Control 32:191-199

Kharrat, S., and A. Jerraya. 2005. Rearing parasitoids by mass production of citrus leafminer larvae, Phyllocnistis citrella (Lepidoptera: Gracillariidae) Entomol. Gen. 28:115-120.

Khyami-Horani H., and M. Ateyyat. 2002. Efficacy of Jordanian isolates of Bacillus thuringiensis against the citrus leafminer, Phyllocnistis citrella Stainton (Lepidoptera: Gracillariidae). Int. J. Pest Manage. 48:297-300.

Klapwijk, J, E.S.Martinez, H. Hoogerbrugge, M. Boogert, and K. den Bolckmans. 2005. The potential of the parasitoid Chrysonotomyia formosa for controlling the tomato leafminer Liriomyza bryoniae in Dutch tomato greenhouses in winter. Bulletin OILB/SROP. 28:155-158

Larraín, P. 2004. Situación de la mosca minadora Liriomyza huidobrensis (Blanchard) en cultivos de la papa del cono sur de América y sus perspectivas de manejo integrado. p. 5-15. En XXI Congreso de la Asociación Latinoamericana de la Papa (ALAP). Valdivia, Chile.

Letourneau, D.K. 1995. associational susceptibility: effects of cropping pattern and fertilizer on malawian bean fly levels. Ecol. Appl. 5:823829.

Lewis, O.T., J. Memmott, J. La Salle, C.H.C. Lyal, C. Whitefoord, and H.J.C. Godfray. 2002. Structure of a diverse tropical forest insectparasitoid community. J. Anim. Ecol. 71:855873.

Lim, U.T., L.M. Zappalà, and A. Hoy. 2006. Prerelease evaluation of Semielacher petiolatus (Hymenoptera: Eulophidae) in quarantine for the control of citrus leafminer: Host discrimination, relative humidity tolerance, and alternative hosts Biol. Control 36:65-73.

Llácer, E., A. Urbaneja, A. Garrido, and J. Jacas. 2006. Temperature requirements may explain why the introduced parasitoid Quadrastichus citrella failed to control Phyllocnistis citrella in Spain. BioControl 51:439-452.

Mafi, S.A., and N. Ohbayashi. 2004. Seasonal prevalence of the citrus leafminer, Phyllocnistis citrella Stainton (Lepidoptera: Gracillariidae) and its parasitoids in controlled and uncontrolled Citrus iyo groves in Ehime Prefecture, Japan. Appl. Entomol. Zool. 39:597-601.

Mafi, S.A., and N. Ohbayashi. 2006. Toxicity of insecticides to the citrus leafminer, Phyllocnistis citrella, and its parasitoids, Chrysocharis pentheus and Sympiesis striatipes (Hymenoptera: Eulophidae) Appl. Entomol. Zool. 41:33-39.

Maier, C.T. 2001. Exotic lepidopteran leafminers in North American apple orchards: rise to prominence, management, and future threats. Biol. Invasions 3:283-293.

Malausa, J.C. 1997. Etat d'avancement de la lutte biologique classique contre la mineuse des agrumes, Phyllocnistis citrella (Stainton) en France. Bulletin OILB/SROP 20:78-80.

Memmott, J., H.C.J. Godfray, and B. Bolton. 1993. Predation and parasitism in a tropical herbivore community. Ecol. Entomol. 18:348-352.

Minkenberg, O., and J. van Lenteren. 1986. The leafminers Liriomyza bryoniae and L. trifolii (Diptera: Agromyzidae), their parasites and host plants: a review. Agricultural University Wagenigen Papers 86:1-50.

Mitsunaga, T., and E. Yano. 2004. The effect of multiple parasitism by an endoparasitoid on several life history traits of leafminer ectoparasitoids Appl. Entomol. Zool. 39:315320. 
Mitsunaga, T. S. Mukawa, T. Shimoda, and Y. Suzuki. 2006. The influence of food supply on the parasitoid against Plutella xylostella L. (Lepidoptera: Yponomeutidae) on the longevity and fecundity of the pea leafminer, Chromatomyia horticola (Goureau) (Diptera: Agromyzidae). Appl. Entomol. Zool. 41:277285.

Motta Miranda, M.M.M., M. Picanço, J.C. Zanuncio, and R.N.C. Guedes. 1998. Ecological life table of Tuta absoluta (Meyrick) (Lepidoptera: Gelechiidae) Biocontrol Sci. Techn. 8:597-606.

Motta Miranda, M.M.M., M. Picanço, J.C. Zanuncio, L. Bacci, and É.M. da Silva. 2005. Impact of integrated pest management on the population of leafminers, fruit borers, and natural enemies in tomato. Ciência Rural 35:204-208.

Murphy, S.T., and J. LaSalle. 1999. Balancing biological control strategies in the IPM of New World invasive Liriomyza leafminers in field vegetables crops. Biocontrol News and Information 20:91-104.

Ode, P.J., and K.M. Heinz. 2002. Host-sizedependent sex ratio theory and improving mass-reared parasitoid sex ratios Biol. Control 24:31-41.

Ohno, K; K. Takesaki, D. Yamaguchi, and H. Takemoto. 1999. Effects of milbemectin acaricide on mortality rate of agromyzid leafminer, Liriomyza trifolii (Burgess), and its larval parasitoid, Diglyphus isaea (Walker) (Hymenoptera: Eulophidae). Japan J. Appl. Entomol. Z. 43:93-97.

Olivera, C.R., and D. Bordat. 1996. Influence of Liriomyza species (Diptera: Agromyzidae) and their host plants, on oviposition by Opius dissitus females (Hymenoptera: Braconidae). Ann. Appl. Biol. 128:399-404.

Palacios, M., J. Tenorio, O. Ortiz, A. Pulcha y R. Gomez. 1995. Implementación y difusión de un programa MIP con énfasis en el control de mosca minadora en el Valle de Tambo, Arequipa, Perú. p. 21. En: Memorias XVII Reunión ALAP, Mérida, Venezuela.

Parkman, P., J.A. Dusky, and V.H. Waddill. 1989. Leafminer and leafminer parasitoid incidence on selected weeds in South Florida. Fla. Entomol. 72: 569-661.

Parrella, M.P. 1987. Biology of Liriomyza. Annu. Rev. Entomol. 32:201-224.

Parrella, M.P., and V.P. Jones. 1987. Development of integrated pest management strategies in floricultural crops. Bull. Entomol. Soc. Am. 33:28-34.

Parrella, M.P., and R. Kaspi. 2005. Effect of abamectin on the leafminer parasitoid Diglyphus isaea. Bulletin OILB/SROP 28:197-200.
Parrella, M.P., G.D. Christie, and K.L. Robb. 1983. Compatibility of Insect Growth Regulators and Chrysocharis parksi (Hymenoptera; Eulophidae) for the control of Liriomyza trifolii (Diptera: Agromyzidae). J. Econ. Entomol. 76:949-951.

Parrella, M.P., J.T. Yost, K.M. Heinz, and G.W. Ferrentino. 1989. Mass rearing of Diglyphus begini (Hymenoptera: Eulophidae) for biological control of Liriomyza trifolii (Diptera: Agromyzidae). J. Econ. Entomol. 82:420-425.

Patel, K.J., D.J. Schuster, and G.H. Smerage. 2003. Density dependent parasitism and host-killing of Liriomyza trifolii (Diptera: Agromyzidae) by Diglyphus intermedius (Hymenoptera: Eulophidae). Fla. Entomol. 86:8-14.

Patel, K.J., and D.J. Schuster. 1991. Temperatura dependent fecundity, longevity, and hostkilling activity of Diglyphus intermedius (Hymenoptera: Eulophidae) on third instars of Liriomyza trifolii (Burgess) (Diptera: Agromyzidae). Environ. Entomol. 20:11951199.

Potter, D.A. 1985. Population regulation of the native holly leafminer, Phytomyza ilicicola Loew (Diptera: Agromyzidae), on American holly. Oecologia 66:499-505.

Powell, W. 1986. Enhancing parasitoid activity in crops. In Insect Parasitoids (J. K. Waage and D. Greathead eds.). Academic Press, London, UK, pp. 319-340.

Preszler, R.W., E.S. Gaylord, and W.J. Boecklen. 1996. Reduced parasitism of a leaf-mining moth on trees with high infection frequencies of an endophytic fungus Oecologia 108:159-166.

Price, J.F., and B.K. Harbaugh. 1981. Effect of cultural practices on Liriomyza, p. 156-185. In: D.J. Schuster (ed.). Proc. I FAS Conf. Biol. Control of Liriomyza leafminers. Buena Vista Lake, Florida, U.S.A.

Price, P., C.E. Bouton, P. Gross, B.A. Mac Pheron, J.N. Thompson, and A.E. Weiss. 1980. Interactions among three trophic levels: the influence of plants on interactions between insect herbivores and natural enemies. Annu. Rev. Ecol. Syst. 11:41-65.

Prijono, D., M. Robinson, A. Rauf, T. Bjorksten, and A.A. Hoffmann. 2004. Toxicity of chemicals commonly used in Indonesian vegetable crops to Liriomyza huidobrensis populations and the Indonesianparasitoids Hemiptarsenusvaricornis, Opius sp., and Gronotoma micromorpha, as well as the Australian parasitoids Hemiptarsenus varicornis and Diglyphus isaea. J. Econ. Entomol. 97:1191-1197.

Queiroz, J.M. 2002. Distribution, survivorship and mortality sources in immature stages 
of the neotropical leaf miner Pachyschelus coeruleipennis Kerremans (Coleoptera: Buprestidae). Braz. J. Biol. 62:69-76.

Quicke, D.L.J. 1997. Parasitic Wasps. Chapman and Hall, London. UK. 470 pp.

Rathman, R.J., M.W. Johnson, J.A. Rosenheim, and B.E. Tabashnik. 1990. Carbamate and pyrethroid resistance in the leafminer parasitoid Diglyphus begini (Hymenoptera: Eulophidae). J. Econ. Entomol. 83:2153-2158.

Rathman, R.J., M.W. Johnson, J.A. Rosenheim, B.E. Tabashnik, and M. Purcell. 1992. Sexual differences in insecticide susceptibility and synergism with piperonyl butoxide in the leafminer parasitoid Diglyphus begini (Hymenoptera: Eulophidae). J. Econ. Entomol. 85:15-20.

Rauf, A., and B.M. Shephard. 1999. Leafminers in vegetables in Indonesia: surveys of host crops, species composition, parasitoids and control practices. p. 25-35. In: Lim, G.S., S.S. Soetikno and W.H. Loke (eds.) Proceedings of a Workshop on Leafminers of Vegetables in Southeast Asia, Tanah Rata, Malaysia.

Reitz, S.R., G.S. Kund, W.G. Carson, P.A. Phillips, and J.T. Trumble. 1999. Economics of reducing insecticide use on celery through low-input pest management strategies Agr. Ecosyst. Environ. 73:185-197.

Rizqi, A., E.B. Nadori, M. Abassi, and M. Nia. 1999. Comparison of three different rearing methods of Ageniaspis citricola, parasitoid of citrus leafminer. p. 247-250. Proceedings 5th World Congress of the International Society of Citrus Nurserymen, Montpellier, France.

Rizzo, M.C. 2003. Tritrophic interactions involving Eulophidparasitoids(Hymenoptera,Eulophidae) of the citrus leafminer Phyllocnistis citrella Stainton. Bulletin OILB/SROP 26:39-51.

Saito, T. 2004. Insecticide susceptibility of the leafminer, Chromatomyia horticola (Goureau) (Diptera: Agromyzidae) Appl. Entomol. Zool. 39:203-208.

Salvo, A. 1996. Diversidad y estructura en comunidades de parasitoides (Hymenoptera: Parasitica) de minadores de hojas (Diptera: Agromyzidae). Tesis Doctoral. Facultad de Ciencias Exactas Físicas y Naturales. Universidad Nacional de Córdoba, Argentina. $355 \mathrm{p}$.

Salvo, A. 2007. Parasitoides de minadores de hojas. En: Biodiversidad de Artrópodos Argentinos Vol II. Debandi G., Roig S. y L. Claps (eds.). Sociedad Entomológica Argentina Ediciones. En Prensa.

Salvo, A., and G.R. Valladares 1996. Intraspecific size variation in polyphagous parasitoids
(Hymenoptera: Parasitica), of leaf miners and its relation to host size. Entomophaga 40:273280.

Salvo, A., and G.R. Valladares. 1999. Parasitoid assemblage size and host ranges in a parasitoid (Hymenoptera)-agromyzid (Diptera) system from Central Argentina. B. Entomol. Res. 89:193-197.

Salvo, A., and G.R. Valladares. 2002. Plant-related intraspecific size variation in three parasitoids (Hymenoptera: Parasitica) of a polyphagous leafminer, Liriomyza huidobrensis (Diptera: Agromyzidae). Environ. Entomol. 30:874-879.

Sato, H. and S. Higashi. 1987. Bionomics of Phyllonorycter (Lepidoptera, Gracillariidae) on Quercus. II. Effects of ants. BioControl 32:53-60.

Schuster, D.J. 1994. Life-stage specic toxicity of insecticides to parasitoids of Liriomyza trifolii (Burgess) (Diptera: Agromyzidae). Int. J. Pest Manage. 40,191-194.

Schuster, D.J., J.P. Gilreath, R.A. Wharton, and P.R. Seymour. 1991. Agromyzidae (Diptera) leafminers and their parasitoids in weeds associated with tomato in Florida. Environ. Entomol. 20:720-723.

Shanower, T.G., J.A. Wightman, A.P. Gutierrez, and G.V. Ranga Rao. 1992. Larval parasitoids and pathogens of the groundnut leaf miner, Aproaerema modicella (Lep.: Gelechiidae), in India. BioControl 37: 419-427

Shen, A.D., Z.Q. Chen, K.J. Luo, S. Miao, and Y.H. Yan. 2003. Toxicity of several insecticides to the larvae, eggs, pupae of the leafminer parasitoid, Diglyphus isaea (Hymenoptera: Eulophidae). Southwest China J. Agric. Sci. 16:69-72.

Shepard, B.M., Samsudin, and A.R. Braun. 1998. Seasonal incidence of Liriomyza huidobrensis (Diptera: Agromyzidae) and its parasitoids on vegetables in Indonesia. Int. J. Pest Manage. 44:43- 47.

Sher, R.B., M.P. Parrella, and H.K. Kaya. 2000. Biological Control of the Leafminer Liriomyza trifolii (Burgess): Implications for Intraguild Predation between Diglyphus begini Ashmead and Steinernema carpocapsae (Weiser). Biol. Control 17:155-163.

Smith, R.M., and J.M. Hardman. 1986. Rates of feeding, oviposition, development and survival of Liriomyza trifolii (Burgess) (Diptera: Agromyzidae) on several weeds. Can. Ent. 118:753-759.

Smith, J.M., and M.A. Hoy. 1995. Rearing methods for Ageniaspis citricola (Hymenoptera: Encyrtidae) and Cirrospilus quadristriatus (Hymenoptera: Eulophidae) released in a classical biological control program for the citrus 
leafminer Phyllocnistis citrella (Lepidoptera: Gracillariidae). Fla. Entomol. 78:600-608.

Spencer, K.A. 1973. Agromyzidae (Diptera) of economic importance. Series Ent. 9. Dr W Junk. The Hague. The Netherlands. 418 pp.

Staley, J.T., S.R. Mortimer, G.J. Masters, M.D. Morecroft, V.K. Brown, and M.E. Taylor. 2006. Drought stress differentially affects leaf-mining species Ecol. Entomol. 31:460-469.

Stiling, P., and D. Simberloff. 1989. Leaf abscission: induced defense against pests or response to damage? Oikos 55:43-49.

Tagami, Y., M. Doi, K. Sugiyama, A. Tatara, and T. Saito. 2006a. Survey of leafminers and their parasitoids to find endosymbionts for improvement of biological control. Biol. Control 38:210-216.

Tagami, Y., M. Doi, K. Sugiyama, A. Tatara, and T. Saito. 2006b. Wolbachia-induced cytoplasmic incompatibility in Liriomyza trifolii and its possible use as a tool in insect pest control. Biol. Control 38:205-209.

Tran, D.H., M. Takagi, and K. Takasu. 2005. Toxicity of selective insecticides to Neochrysocharis formosa (Westwood) (Hymenoptera: Eulophidae), a parasitoid of the American serpentine leafminer Liriomyza trifolii (Burgess) (Diptera: Agrizomydae). J. Fac. Agric. Kyushu Univ. 50:109-118.

Urbaneja, A., E. Llácer, A. Garrido, and J. Jacas. 2000. Indigenous Natural Enemies Associated with Phyllocnistis citrella (Lepidoptera: Gracillariidae) in Eastern Spain. Biol. Control 18:199-207.

Urbaneja, A., E. Llácer, A. Garrido, and J. Jacas. 2001. Effect of variable photoperiod on development and survival of Cirrospilus sp. nr. lyncus (Hymenoptera: Eulophidae), an ectoparasitoid of Phyllocnistis citrella (Lepidoptera: Gracillariidae). Fla. Entomol. 84:305-307.

Urbaneja, A., E. Llácer, A. Garrido, and J. Jacas. 2003. Interspecific competition between two ectoparasitoids of Phyllocnistis citrella (Lepidoptera: Gracillariidae): Cirrospilus brevis and the exotic Quadrastichus sp. (Hymenoptera: Eulophidae). Biol. Control 28:243-250.

Uygun, N., N.Z. Elekcioglu, L. Erkilic, I. Karaca, and U. Kersting. 1997. Studies on biological control of Phyllocnistis citrella Stainton (Lep., Gracillariidae) in Turkey. Bulletin OILB/SROP 20:96-101.

Valladares, G.R. Agromyzidae. 2007. En Biodiversidad de Artrópodos Argentinos Vol II. Debandi G., Roig S. y L. Claps (eds.) Sociedad Entomológica Argentina Ediciones. En prensa.
Valladares, G.R., and Lawton, J.H. 1991. Host-plant selection in the holly leaf-miner: does mother know best? J. Anim. Ecol. 60:227-240.

Valladares G.R., and A. Salvo. 1999. Insect-plant food webs could provide new clues for Pest Management. Environ. Entomol. 28:539-544.

van der Linden, A. 1992. Phytomyza caulinaris Hering, an alternative host for the development of an open rearing system for parasitoids of Liriomyza species. P. Sec. Exp. Appl. Entomol. Neth. Ent. Soc. 3:31-39.

van der Linden, A. 1994. Can biological control of Liriomyza spp in glasshouse crops be improved?. Med. Fac. Landbouww. Univ. Gent. 59:297-303.

van Driesche, R.G., J.L. Mason, S.E. Wright, and R.J. Prokopy. 1998. Effect of reduced insecticide and fungicide use on parasitism of leafminers (Phyllonorycter spp) (Lepidoptera: Gracillariidae) in commercial apple orchards. Environ. Entomol. 27:578-582.

van Lenteren J.C., and W.A. Overholt. 1994. Ecology and Integrated Pest Management. Insect Sci. Appl. 6:557-582.

van Lenteren, J.C., and J. Woets. 1988. Biological and integrated control in greenhouses. Annu. Rev. Entomol. 33:239-269.

van Mele, P., and J.C. van Lenteren. 2002. Survey of current crop management practices in a mixedricefield landscape, Mekong Delta, Vietnam potential of habitat manipulation for improved control of citrus leafminer and citrus red mite. Agr. Ecosys. Environ. 88:35-48.

Vercher, R., J. Costa-Comelles, C. Marzal, and F. Garcia-Mari. 2005. Recruitment of native parasitoid species by the invading leafminer Phyllocnistis citrella (Lepidoptera: Gracillariidae) on citrus in Spain. Environ. Entomol. 34:1129-1138.

Villanueva-Jiménez, J.A., and M.A. Hoy. 1998. Toxicity of pesticides to the citrus leafminer and its parasitoid Ageniaspis citricola evaluated to assess their suitability for an IPM program in citrus nurseries. BioControl 43:357-388.

Vincent, C., B. Rancourt, and O. Carisse. 2004. Apple leaf shredding as a non-chemical tool to manage apple scab and spotted tentiform leafminer. Agr. Ecosys. Environ. 104:595-604.

Wang, L., M. You, J. Wang, and Q. Wu Q. 1999. Niches of citrus leafminer and its natural enemies. J. Fujian Agric. Univ. 28:319-324.

Weintraub, P.G. 1999. Effects of cyromazine and abamectin on the leafminer, Liriomyza huidobrensis and its parasitoid, Diglyphus isaea in celery Ann. Appl. Biol. 135:547-554.

Weintraub, P.G., and A.R. Horowitz. 1998. Effects of translaminar versus conventional 
insecticides on Liriomyza huidobrensis (Diptera: Agromyzidae) and Diglyphus isaea (Hymenoptera: Eulophidae) populations in celery. J. Econ. Entomol. 91:1180-1185.

West, S.A., J.M. Cook, J.H. Werren, and H.C.J. Godfray. 1998. Wolbachia in two insect hotsparasitoid communities. Mol. Ecol 7:1457-1465.

Wu T.K. and K.S. Lin. 1998. Influence of green lacewing, Mallada basalis (Walker) (Neuroptera: Chrysopidae), on parasitoids of citrus leafminer, Phyllocnistis citrella Stainton (Lepidoptera: Phyllocnistidae). Chin. J. Entomol. 18:13-25.

Xiao, Y., J.A. Qureshi, and P.A. Stansly. 2007. Contribution of predation and parasitism to mortality of citrus leafminer Phyllocnistis citrella Stainton (Lepidoptera: Gracillariidae) populations in Florida. Biol. Control 40:396-404.
Yarnes, C.T., and W.J. Boecklen. 2006. Abiotic mosaics affect seasonal variation of plant resources and influence the performance and mortality of a leaf-miner in Gambel's oak (Quercus gambelii Nutt.). Ecol. Res. 21:157163.

Yoder, J.A., and M.A. Hoy. 1998. Differences in water relations among the citrus leafminer and two different populations of its parasitoid inhabiting the same apparent microhabitat. Entomol. Exp. Appl. 89:169-173.

Zappala, L., and M.A. Hoy. 2004. Reproductive strategies and parasitization behavior of Ageniaspis citricola, a parasitoid of the citrus leafminer Phyllocnistis citrella. Entomol. Exp. Appl. 113:135-143. 